\section{P54 iCook 4-H: 0 to 24-Month Accelerometer- Derived Physical Activity and Sedentary Time in Youth}

Emily Hofer, BS, RDN, emily.hofer@jacks.sdstate.edu, South Dakota State University, Box 2203, Department of Health \& Nutritional Sciences, Brookings, SD 57007; K. Kattelmann, PhD, RDN, LN, FAND; C. Merfeld, BS; J. Meendering, PhD, EP-C; M. Olfert, DrPH, MS, RDN, LDN, West Virginia University; R. Hagedorn, BS; S. Colby, PhD, $R D$, University of Tennessee; L. Franzen-Castle, $M S, R D$, $P h D$, University of Nebraska-Lincoln; D. Mathews, PhD, $R D$, University of Maine; $A$. White, $P h D, R D$

Objective: To assess accelerometer-derived physical activity (PA) and sedentary time (ST) from 0 to 24-months in iCook 4-H youth.

Design, Setting, Participants, and Intervention: iCook 4 -H was a 5-state, randomized control-treatment family-based childhood obesity prevention intervention promoting cooking, eating and playing together. Youth, 9-10 years old, and their main meal preparer participated in the 14-week program followed by monthly newsletters and bi-yearly booster sessions until 24-months. PA and ST were determined for 124 (Intervention=88; Control=36) youth.

Outcome Measures and Analysis: Youth wore an Actigraph GT3X+ accelerometer for 7 days at 0, 4, 12, and 24months. Mean daily minutes of ST, light PA (LPA), moderate PA (MPA), vigorous PA (VPA) and moderate-to-vigorous PA (MVPA) were evaluated during waking hours. Between and within group differences from 0 to 24 months were determined using linear mixed models ( $\mathrm{R}, 3.2 .3$, Vienna, Austria, 2015); $\mathrm{p} \leq 0.05$.

Results: There were no between group differences in PA or ST at any time-point. PA at all intensity levels decreased and ST increased for both groups from 0 to 24-months $(p \leq 0.001)$. The percent of participants meeting the PA guidelines (60 minutes of MVPA per day) decreased from $0 \quad(60.8 \%$ intervention, $84.5 \%$ control) to 24 -months (16\% intervention, 0\% control).

Conclusion and Implications: iCook 4-H was a multicomponent program following 9-10 year old youth for 24-months that focused on cooking skills, mealtime behavior and conversation, and enhancing PA through daily activities. Greater emphasis on developing PA skills, changing environmental factors, and increasing PA both in and after school may be needed to prevent the decrease in PA that occurs as children age into adolescence.

Funding: USDA

\section{P55 Efficacies of Color Your Plate Nutrition Education Curriculum in Senior Center Congregate Meal Sites}

Alyson Humphrey, BS, alyson.humphrey@uky.edu, University of Kentucky, 420 Redding Road, Lexington, KY 40517

Objective: Nutrition education classes at senior centers have the opportunity to be an effective tool to increase fruit and vegetable intake in older adults. The Color Your Plate nutrition education program was developed to help older adults overcome perceived barriers to fruits and vegetable consumption and increase motivation to eat recommended amounts of fruits and vegetables.

Design, Setting, and Participants: Older adults regularly attending congregate meals at four senior centers were recruited to participate in this study. At two experimental sites, seniors engaged in a five-session nutrition education program offered biweekly at the senior center based off the University of Georgia Live Well Curriculum. Control participants received print nutrition education materials, but no classes.

Outcome Measures and Analysis: All participants were interviewed by trained study personnel pre-nutrition education, immediately at the end of the program, and at 2 and 4 months post-intervention.

Results: Sixty-five seniors (18.5\% males, $81.5 \%$ females) ages $75.6 \pm 8.0$ years participated. For those seniors engaged in nutrition education classes, there was a significant increase in motivation to consume more fruits and vegetables post-intervention versus pre-intervention $(\mathrm{p}=0.003)$. The main barriers to fruit and vegetable consumption were lack of availability when not in season $(67.2 \%)$, dislike for the taste (42.2\%), and lack of adequate storage space $(31.1 \%)$. The nutrition education program did not have a significant impact on decreasing barriers to fruit and vegetable consumption.

Conclusions and Implications: The five-lesson Color Your Plate nutrition education program increased motivation of older adults to consume more fruits and vegetables. In the future, the program could greater emphasize strategies to overcoming barriers to fruit and vegetable consumption.

Funding: None

\section{P56 Perspectives Toward Partnering With Faith Organizations for Health Programming} Kimberlee Kinney, Student, BS, PhD, skim13@vt.edu, Virginia Tech, Department of Human Nutrition, Foods and Exercise, Wallace Hall, Blacksburg, VA 24061; E. Serrano, PhD; J. Midkiff; J. Stevens; K. Hosig, $\mathrm{PhD}, \mathrm{MPH}, \mathrm{RD}$

Objective: To explore the perspectives, capacity, and readiness of Supplemental Nutrition Assistance Program Education (SNAP-Ed) Extension Agents from Virginia Cooperative Extension (VCE) in partnering with faithbased organizations (FBO) to deliver nutrition-education health programming.

Design, Setting, and Participants: A mixed-methods study with online surveys and individual telephone interviews with current and former VCE SNAP-Ed Agents $(n=10)$ was conducted to explore Agents' previous experiences and future interest towards partnering and working with FBOs.

Outcome Measures and Analysis: Outcomes included agent's previous experience(s), current opportunities, future interest working with FBOs, barriers and facilitators

Continued on page S31 